\title{
Nonlinear Feedback System Stability via Coprime Factorization Analysis*
}

\author{
A.D.B. Paice ${ }^{\dagger}$ \\ J.B. Moore ${ }^{\dagger}$ \\ R. Horowitz ${ }^{\dagger}$
}

\begin{abstract}
In this paper right coprime factorization results are derived for : general class of nonlinear plants and stabilizing feedback controllers Both input-output descriptions and state space realizations of the plant and controller are used.

It is first shown that if there exist stable right coprime factorizations for the plant and controller, and if a certain matrix of nonlinear operators has a stable inverse then the feedback system is well-posed and internally stable. The links between the right and left coprime factorizations for a stable plant controller pair will be explored for this purpose. A generalization of the notion of linear fractional maps is explored as a means of characterizing the class of plants stabilized by this controller, and dually classes of controllers which stabilize the plant.

It is then shown how to apply this theory to nonlinear plants which have a state space realization of a given form. It is also shown that if there exists a stabilizing state feedback for a plant in the class of interest, then there exists a right coprime factorization for the plant. Additionally if there exists a stabilizing output injection, then there will exist a stabilizing controller with a right coprime factorization. An important assumption in this work is to assume that the plant and controller have the same initial conditions, an approach is developed to allow for the stabilization of the plant by a controller with a different initial condition. A similar approach may also be used to stabilize a plant which has unmodeled dynamics.

These results, of course, specialize to familiar linear system ones, and just as such linear systems results have had a wide application in robust and adaptive control system design, it is believed that the results developed here will facilitate the development of corresponding nonlinear robust and adaptive control system design.
\end{abstract}

* Received June 19, 1991; received in final form September 16, 1991.

'Work partially supported by DSTO Australia and Boeing (BCAC). 


\section{A.D.B. PAICE, J.B. MOORE, AND R. HOROWITZ}

Key words: coprime factorizations, input-output, state space

\section{Introduction}

The theory of coprime factorizations and applications to the stabilization of linear systems is well understood [23]. This theory has proven useful for giving robust design procedures [2] and in providing a venue for the implementation of adaptive control [19]. More recently there has been some interest in generalizing these results to the nonlinear case. An important challenge is to set the foundations for a general approach to the feedback stabilization of nonlinear systems, while paralleling the linear systems development as much as possible. Although experience has shown that in many instances the nonlinear theory is surprisingly close to the linear theory, it would be naive to expect to prove nonlinear generalizations of all linear systems results. It seems crucial to some of the linear systems results is in the application of the principle of superposition, which is of course not permitted for nonlinear systems.

Some of the first investigations into the field of nonlinear factorization analysis were carried out by Hammer in his papers $[3,4,5,6,7]$. An inputoutput approach along with set-theoretic techniques were used to develop a pre- and post-compensator pair to stabilize a given plant. Tay [18] used this framework to develop the class of all pre-, post-compensator pairs that stabilize a given plant. By using the concept of differential boundedness, introduced in [6], Paice [14] was able to generate the class of all controllers bounded-input stabilizing a given plant. This was further developed in [15] to generate a result giving the classes of all plants stabilized by a given controller, and to give some robust stabilization results for systems within that framework. Note that these papers worked mainly with the left factorizations of a given plant, controller pair, and worked within a purely input-output framework.

At the same time the theory using the right factorizations was being investigated, notably by Desoer [1] and Verma [20,21]. This work was also conducted from within an input-output framework. The results obtained in this work were similar to those obtained through the left factorization approach, but linearity assumptions had to be invoked, for either the plant or controller.

More recently there has been some interest in finding state space realizations of the factorizations for nonlinear systems. Sontag [16] presented results giving a right factorization for a class of nonlinear plants, and linked them to the problem of finding a smooth stabilizing state feedback map for the plant of interest. Krener [11] presented results showing that right and left factorizations could be obtained for nonlinear plants with controller and observer normal forms. Of particular interest here was the augmenta- 


\section{COPRIME FACTORIZATION ANALYSIS}

tion of the plant by a unity feedthrough term, which appeared necessary to obtain a left factorization. This is required also in the companion work by Moore and Irlicht [12], in which a factorization theory is developed for a quite general form of nonlinear plants, giving right factorizations, and left factorizations for an augmented version of the plant. In [22] Verma presented a construction of the right coprime factorization of a ger eral continuous time nonlinear plant, while in [8] Hammer gives a construction for discrete time systems.

Throughout this previous and present work it has become apparent ihat by making very few simplifying assumptions, a framework which clo sely mimics the linear factorization theory may be developed. It has become of interest to see in what way the linear theory is dependent on the linearity assumption, and to see how extensive a theory may be developed while making only the minimum of assumptions on the class of plants urder consideration. It is the purpose of this paper to examine this question

Both the input-output framework and state space realizations of the plant and controller shall be considered. First, from an input-output point of view, general results are derived showing the relationship between the inverse of a matrix composed of right coprime factorizations of the plant and controller, and the well-posedness and stability of the feedback system. Dual formulations, in terms of using the left factorizations shall $:$ lso be considered. A generalization of linear fractional maps shall then be derived, offering a method of deriving the class of plants stabilized by a gi ren controller, or the class of controllers stabilizing the plant. Then, working with a general state space description of the plant, right factorizations and a stabilizing controller for a given plant are derived, and some of the previous results derived are applied giving an approach to the stabilization problem which allows for differing initial conditions and unmode ed dynamics. Specifically the paper is organised as follows.

Section 2 develops some of the basic concepts of factorization analysis, and introduces the two main schemes, right and left factorizations. In Section 3 a nonlinear generalization of linear fractional mappings is int $/ 0-$ duced and explored. In Section 4 we derive right factorizations for a plant for which there exists a stabilizing state feedback map. A controller is also designed, based on the idea of constructing a stable state estimator for $t$ le plant, a right factorization for this controller is also presented. Throu,, $\mathrm{h}$ the use of some of the results of Section 2 it is shown that these factoriz ations are coprime and that the plant controller feedback loop is stable. A $\mathrm{n}$ approach to the stabilization of a plant with different initial conditions $: 0$ those of the controller through the use of Theorem 3.2 is also presented. In Section 5 a special form of the nonlinear system is considered as a means if obtaining left factorizations. The main result of [15] may then be applit $\mathrm{d}$ to give the class of all bounded input stable plants and controllers. 


\section{Preliminaries}

Consider the problem of stabilizing a nonlinear plant $G: C(\mathcal{U}) \mapsto C(\mathcal{Y})$ by a controller $K: C(\mathcal{Y}) \mapsto C(\mathcal{U})$, such that the closed-loop system shown in Figure 1 is stable. For convenience we denote this feedback control system $\{G, K\}$. Continuous time systems with real input spaces are considered. Given a real vector space $\mathcal{X}$, the space $C(\mathcal{X})$ is the space of continuous functions with continuous first derivative, mapping from some open interval of $\mathcal{R}$ to $\mathcal{X}$. Note that the results obtained apply equally to discrete time systems as to continuous time systems.

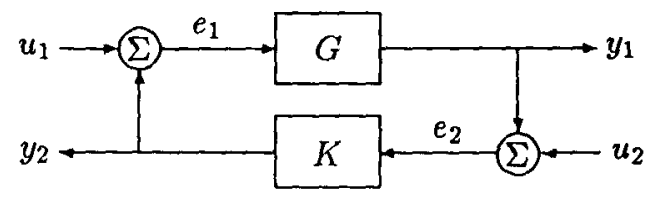

Figure 1: The feedback system $\{G, K\}$.

Definition 2.1 [Well-posedness] The system $\{G, K\}$ is well-posed if the closed-loop system input-output operator from $u_{1}, u_{2}$ to $e_{1}, e_{2}$, namely $\left[\begin{array}{cc}I & -K \\ -G & I\end{array}\right]^{-1}$, exists.

In the sequel only those systems which are well-posed shall be considered.

In the following two sections the stability problem for the system $\{G, K\}$ is developed from an input-output point of view so as to keep the discussion as general as possible. We partition the space $C(\mathcal{X})$ into two subspaces, $C_{b}(\mathcal{X})$ and $C_{u}(\mathcal{X})$. The former consists of all signals in $C(\mathcal{X})$ which are bounded, or stable, while the latter consists of all signals in $C(\mathcal{X})$ which are unbounded. The signal $x \in C(\mathcal{X})$ is said to be bounded when $\|x\|$ is finite, for some norm $\|\cdot\|$.

Definition 2.2 [BIBO Stability] $A$ map $F: C(\mathcal{U}) \mapsto C(\mathcal{Y})$ is said to be bounded-input, bounded-output stable (BIBO stable) when the image of $C_{b}(\mathcal{U})$ under $F$ is contained in $C_{b}(\mathcal{Y})$.

Definition 2.3 [Internal Stability] The system $\{G, K\}$, assumed wellposed, is said to be internally stable iff for all bounded-inputs $u_{1}, u_{2}$ the outputs $y_{1}, y_{2}$ and $e_{1}, e_{2}$ are bounded. This is equivalent to

$$
\left[\begin{array}{cc}
I & -K \\
-G & I
\end{array}\right]^{-1} \text { is BIBO stable. }
$$




\section{COPRIME FACTORIZATION ANALYSIS}

Definition 2.4 [Bounded-Input Stability] The system $\{G, K\}$, as:umed well-posed, is said to be $\varepsilon_{1}, \varepsilon_{2}$ bounded-input stable iff for all inputs $u_{1} \mid<$ $\varepsilon_{1},\left|u_{2}\right|<\varepsilon_{2}$ the outputs $y_{1}, y_{2}$ and $e_{1}, e_{2}$ are bounded.

Note that internal stability is a stronger condition than bounded-input stability.

A factorization approach to the stabilization of the plant $G$ is taken, in analogy with the linear theory of Youla-Kucera parameterizations The following definitions are taken from [15], and have been developed from the point of view of preventing the nonlinear equivalent of unstable polezero cancellations, and thus for linear systems specialize to right half slane coprimeness.

Definition 2.5 [Right coprimeness] Let $M, N$ be a right factorization for $G: C(\mathcal{U}) \rightarrow C(\mathcal{Y})$

$$
\begin{array}{ll}
G=N M^{-1}, & \left.N: C\left(\mathcal{S}_{\mathrm{r}}\right) \rightarrow C(\mathcal{Y})\right) \\
& M: C\left(\mathcal{S}_{\mathrm{r}}\right) \rightarrow C(\mathcal{U})
\end{array}
$$

where $M$ and $N$ are $B I B O$ stable mappings from the factorization space $C\left(\mathcal{S}_{r}\right)$ to the input and output spaces. Then $M, N$ is a right coprime factorization of $\mathrm{G}(\mathrm{rcf})$ iff for all unbounded inputs $s \in C_{u}\left(\mathcal{S}_{\mathrm{r}}\right)$, Ms or $\Lambda s$ is unbounded.

Definition 2.6 [Left coprimeness] Let $\tilde{M}, \tilde{N}$ be a left factorization for $G: C(\mathcal{U}) \rightarrow C(\mathcal{Y})$

$$
G=\tilde{M}^{-1} \tilde{N} \quad, \quad \begin{aligned}
& \tilde{N}: C(\mathcal{U}) \rightarrow C\left(\mathcal{S}_{1}\right) \\
& \tilde{M}: C(\mathcal{Y}) \rightarrow C\left(\mathcal{S}_{1}\right)
\end{aligned}
$$

where $\tilde{M}, \tilde{N}$ are $B I B O$ stable mappings from the input and output spcces to the factorization space $C\left(\mathcal{S}_{1}\right)$. Then $\tilde{M}, \tilde{N}$ is a left coprime factorization of $\mathrm{G}$ (lcf) iff the set of all unbounded $u \in C_{u}(\mathcal{U})$ such that $G u$ is boun led and $\tilde{N} u$ is bounded is the empty set, $\emptyset$. In other words, for all bouniled $\zeta \in C_{b}\left(\mathcal{S}_{1}\right), \tilde{M}^{-1} \zeta$ is bounded or $\{u: \tilde{N} u=\zeta\}$ is bounded, which is an explicit dual statement of the definition for right coprimeness.

To prove coprimeness of a factorization, the definition may be test $2 \mathrm{~d}$ directly, or the following lemma may be used.

Lemma 2.1 Given a stable right factorization of $G$, as in (2.2), suppose that there exists a $B I B O$ stable mapping $L: C(\mathcal{U}) \times C(\mathcal{Y}) \mapsto C\left(\mathcal{S}_{\mathrm{r}}\right)$ such that

$$
L\left[\begin{array}{c}
M \\
N
\end{array}\right]=Z, \quad Z \text { unimodular. }
$$

Then $G=N M^{-1}$ is a right coprime factorization for $G$. 
Proof: Consider $L: C(\mathcal{U}) \times C(\mathcal{Y}) \mapsto C\left(\mathcal{S}_{\mathrm{r}}\right)$ a BIBO stable mapping which satisfies (2.4). Suppose that $N, M$ is not a coprime factorization for $G$. Then there exists an unbounded $s \in C\left(\mathcal{S}_{\mathrm{r}}\right)$ such that $M s$ and $N s$ are both bounded. As $L$ is BIBO, $L\left(\begin{array}{c}M_{s} \\ N_{s}\end{array}\right)=Z s$ is bounded, however as $Z$ is unimodular, $Z s$ is unbounded. This gives a contradiction, proving the result.

Remark. In the case that $L=\left[\begin{array}{ll}L_{1} & L_{2}\end{array}\right]$, this lemma specializes to Lemma 2.1 of [15].

If the system $\{G, K\}$ is well-posed and stable, assume that in addition to having stable coprime descriptions for $G$ as in (2.2) and (2.3) there are factorizations for $K: C(\mathcal{Y}) \mapsto C(\mathcal{U})$. i.e

$$
\begin{array}{ll}
K=U V^{-1}, & U: C\left(\mathcal{S}_{\mathrm{r}}\right) \rightarrow C(\mathcal{U}) \\
& V: C\left(\mathcal{S}_{1}\right) \rightarrow C(\mathcal{Y}) \\
K=\tilde{V}^{-1} \tilde{U}, & , \quad \tilde{U}: C(\mathcal{Y}) \rightarrow C\left(\mathcal{S}_{\mathrm{r}}\right) \\
& \quad \tilde{V}: C(\mathcal{Y}) \rightarrow C\left(\mathcal{S}_{1}\right)
\end{array}
$$

where $V, U, \tilde{V}, \tilde{U}$ are BIBO stable operators and $C\left(\mathcal{S}_{1}\right)$ and $C\left(\mathcal{S}_{\mathrm{r}}\right)$ are the factorization spaces.

In subsequent sections it will be useful to consider the stability and well-posedness of the system $\{G, K\}$ via the stability and existence of the operators $\left[\begin{array}{cc}M & -U \\ -N & V\end{array}\right]^{-1}$ and $\left[\begin{array}{cc}\tilde{V} & -\bar{U} \\ -\tilde{N} & \bar{M}\end{array}\right]^{-1}$. In the former case where $r c f$ 's of $G, K$ are used, the relationship is straightforward, whereas the relationship is more complicated for $l c f$ 's.

\section{Right Coprime Factorization Results}

The following theorem and lemma show well-posedness and coprimeness are necessary and sufficient for the existence and stability of the operator inverse.

Theorem 2.1 Given $\{G, K\}$, and $G=N M^{-1}$ and $K=U V^{-1}$ rcf's as in (2.2) and (2.5), then $\{G, K\}$ is well-posed iff

$$
\left[\begin{array}{cc}
M & -U \\
-N & V
\end{array}\right]^{-1} \text { exists }
$$

and is internally stable iff

$$
\left[\begin{array}{cc}
M & -U \\
-N & V
\end{array}\right]^{-1} \text { is BIBO stable. }
$$




\section{COPRIME FACTORIZATION ANALYSIS}

Proof: First we note that

$$
\begin{aligned}
{\left[\begin{array}{cc}
I & -K \\
-G & I
\end{array}\right]^{-1} } & =\left[\begin{array}{cc}
I & -U V^{-1} \\
-N M^{-1} & I
\end{array}\right]^{-1} \\
& =\left\{\left[\begin{array}{cc}
M & -U \\
-N & V
\end{array}\right]\left[\begin{array}{cc}
M^{-1} & 0 \\
0 & V^{-1}
\end{array}\right]\right\}^{-1} \\
& =\left[\begin{array}{cc}
M & 0 \\
0 & V
\end{array}\right]\left[\begin{array}{cc}
M & -U \\
-N & V
\end{array}\right]^{-1}
\end{aligned}
$$

It is straightforward to see (2.7) holds iff $\{G, K\}$ is well-posed.

$(\nLeftarrow)$ Suppose that $(2.8)$ holds, then for all $a, b$ bounded we define $c, d$ as follows:

$$
\left(\begin{array}{l}
c \\
d
\end{array}\right)=\left[\begin{array}{cc}
M & -U \\
-N & V
\end{array}\right]^{-1}\left(\begin{array}{l}
a \\
b
\end{array}\right)
$$

$c, d$ are bounded. Hence, by (2.9),

$$
\left[\begin{array}{cc}
I & -K \\
-G & I
\end{array}\right]^{-1}\left(\begin{array}{l}
a \\
b
\end{array}\right)=\left[\begin{array}{cc}
M & 0 \\
0 & V
\end{array}\right]\left(\begin{array}{l}
c \\
d
\end{array}\right) .
$$

Under (2.2), (2.5) $M$ and $V$ are BIBO stable. Hence $M c$ and $V d$ are BIBO thus showing that the system inverse operator exists and is BIBO.

$(\Rightarrow)$ Suppose that $\{G, K\}$ is well posed and stable and that $G=\| M^{-1}$ and $K=U V^{-1}$ are stable rcf's. Let

$$
\left(\begin{array}{l}
e \\
f
\end{array}\right)=\left[\begin{array}{cc}
I & -K \\
-G & I
\end{array}\right]^{-1}\left(\begin{array}{l}
a \\
b
\end{array}\right)
$$

then for all $a, b$ bounded, we have $e, f$ bounded. Define $c, d$ as in (2.10), note that as $a, b$ and $e, f$ are bounded, the following equations hold

$$
\begin{aligned}
& \left(\begin{array}{l}
a \\
b
\end{array}\right)=\left[\begin{array}{c}
M c-U d \\
-N c+V d
\end{array}\right] \\
& \left(\begin{array}{l}
e \\
f
\end{array}\right)=\left(\begin{array}{c}
M c \\
V d
\end{array}\right) .
\end{aligned}
$$

As $e$ is bounded $M c$ is bounded, and since $a$ and $M c$ are bounded, i $d$ is bounded. Similarly, as $b$ and $f$ are bounded, $V d$ and $N c$ are bounded. By coprimeness of $N M^{-1}$, since $N c$ and $M c$ are both bounded, $c$ is bour ded. Similarly, by coprimeness of $U V^{-1}, d$ is bounded. This completes the proof.

Hence the stability and well-posedness of the system depends on the existence and stability of the operator $\left[\begin{array}{cc}M & -U \\ -N & V\end{array}\right]^{-1}$. In fact the relai,ionship is somewhat stronger, coprimeness also results from the stability of this operator, as is explored by the following lemma. 


\section{A.D.B. PAICE, J.B. MOORE, AND R. HOROWITZ}

Lemma 2.2 Suppose we have $G=N M^{-1}$ and $K=U V^{-1}$, such that the operators $M, N, U, V$ are $B I B O$ stable. Then these are rcfs for $G$ and $K$ if they satisfy (2.8).

Proof: Since the matrix inverse is stable we require that unbounded inputs yield unbounded inputs. Consider $x$ an unbounded signal, and consider the action of the system as follows.

$$
\left[\begin{array}{cc}
M & -U \\
-N & V
\end{array}\right]\left(\begin{array}{l}
x \\
0
\end{array}\right)=\left[\begin{array}{c}
M x-U 0 \\
-N x-V 0
\end{array}\right]
$$

As $x$ is unbounded, the output is also unbounded. Thus we must have $M x$ or $N x$ unbounded, giving coprimeness of $M, N$. Considering the action of $\left[\begin{array}{cc}M & -U \\ -N & V\end{array}\right]\left(\begin{array}{l}0 \\ y\end{array}\right)$ for $y$ unbounded gives coprimeness of $U, V$.

\section{Left Coprime Factorization Results}

The relationship between the operator $\left[\begin{array}{cc}\tilde{V} & -\tilde{U} \\ -\tilde{N} & \tilde{M}\end{array}\right]^{-1}$ and the stability and well-posedness of $\{G, K\}$ is not so clear. In the linear case we have that this operator is stable iff the system $\{G, K\}$ is well-posed and stable. The only similar result existing for the nonlinear case at present is in the remarks to Lemma 4.1 of [15], which states that if the maps $\tilde{M}, \tilde{N}, \tilde{U}, \tilde{V}$ are differentially bounded we have bounded-input stability of the operator iff $\{G, K\}$ is bounded-input stable. Differential boundedness is defined as follows.

Definition 2.7 An operator $F: C(\mathcal{X}) \mapsto C(\mathcal{Y})$ is said to be differentially bounded by $\theta_{F}, \varepsilon_{F}$ iff for all signals $a_{1}, a_{2} \in C(\mathcal{X})$, if $\left|a_{1}-a_{2}\right|<\varepsilon_{F}$ then $\left|a_{1}-a_{2}\right|<\theta$.

More general results are elusive, although the following result may be obtained.

Lemma 2.3 Suppose that for $G$ and $K$, we have Icf's as in (2.3), (2.6), then if

$$
\left[\begin{array}{cc}
\tilde{V} & -\tilde{U} \\
-\tilde{N} & \tilde{M}
\end{array}\right]^{-1} \text { exists, }
$$

then it is necessary for the operators $(\tilde{V}-\tilde{U} G)^{-1}$ and $(\tilde{M}-\tilde{N} K)^{-1}$ to exist. Moreover the operators $(\tilde{V}-\tilde{U} G)^{-1}$ and $(\tilde{M}-\tilde{N} K)^{-1}$ are stable if

$$
\left[\begin{array}{cc}
\tilde{V} & -\tilde{U} \\
-\tilde{N} & \tilde{M}
\end{array}\right]^{-1} \text { is stable. }
$$




\section{COPRIME FACTORIZATION ANALYSIS}

Proof: Consider the inputs $\left(\begin{array}{l}x \\ 0\end{array}\right)$ and $\left(\begin{array}{l}0 \\ y\end{array}\right)$ to $(2.16)$. Then we have

$$
\begin{aligned}
& \left(\begin{array}{l}
x \\
0
\end{array}\right)=\left[\begin{array}{cc}
\tilde{V} & -\tilde{U} \\
-\tilde{N} & \tilde{M}
\end{array}\right]\left(\begin{array}{c}
a_{x} \\
b_{x}
\end{array}\right) \\
& =\left(\begin{array}{c}
\tilde{V} a_{x}-\tilde{U} b_{x} \\
-\tilde{N} a_{x}+\tilde{M} b_{x}
\end{array}\right) \\
& \left(\begin{array}{c}
a_{x} \\
b_{x}
\end{array}\right)=\left(\begin{array}{c}
G(\tilde{V}-\tilde{U} G)^{-1} x \\
(\tilde{V}-\tilde{U} G)^{-1} x
\end{array}\right) \\
& \left(\begin{array}{l}
0 \\
y
\end{array}\right)=\left[\begin{array}{cc}
\tilde{V} & -\tilde{U} \\
-\tilde{N} & \tilde{M}
\end{array}\right]\left(\begin{array}{l}
a_{y} \\
b_{y}
\end{array}\right) \\
& =\left(\begin{array}{c}
\tilde{V} a_{y}-\tilde{U} b_{y} \\
-\tilde{N} a_{y}+\tilde{M} b_{y}
\end{array}\right) \\
& \left(\begin{array}{l}
a_{y} \\
b_{y}
\end{array}\right)=\left(\begin{array}{c}
(\tilde{M}-\tilde{N} K)^{-1} y \\
K(\tilde{M}-\tilde{N} K)^{-1} y
\end{array}\right)
\end{aligned}
$$

The results of the lemma follow from these equations.

\section{Remarks}

1. The existence of $(\tilde{V}-\tilde{U} G)^{-1}$ corresponds to the scheme of [6] in which the plant $G$ is stabilized by a pre- and feedback-compensator pair $\tilde{V}^{-1}, \tilde{U}$. Without the differential boundedness assumptions mentioned above it is not clear how this result relates to the well-posedness of the system $\{G, K\}$.

2. In the case that there exist lcf's for $G$ and $K$ in which the ope:ators $\tilde{V}, \tilde{M}$ are linear it is possible to reproduce the linear theory or left coprime factorizations. Currently there does not appear to be any way to link stability and well-posedness of $\{G, K\}$ to equaticns $(2.16),(2.17)$ without this linearity assumption.

\section{Fractional Maps}

Let us now study a nonlinear equivalent of the idea of linear fractional maps. The idea is to develop a framework to characterize the class of stabilizing controllers for a given plant, and the class of plants stabiliz; $d$ by a given controller. The first result concerns left coprime factorizatio is for $G_{S}$, "stabilized" by $K$ in a restricted sense.

Theorem 3.1 Consider a well-posed and internally stable system $\left\{G, F^{\prime}\right\}$ with left coprime factorizations (2.3), (2.6). Consider also any plant ('s 
such that

$$
\left(\tilde{V}-\tilde{U} G_{S}\right)^{-1} \text { exists }
$$

then $G_{S}$ has a right factorization

$$
G_{S}=N_{S} M_{S}^{-1} \quad,\left[\begin{array}{c}
M_{S} \\
N_{S}
\end{array}\right]=\left[\begin{array}{c}
I \\
G_{S}
\end{array}\right]\left(\tilde{V}-\tilde{U} G_{S}\right)^{-1}
$$

and satisfies the Bezout identity

$$
\tilde{V} M_{S}-\tilde{U} N_{S}=I
$$

If $M_{S}, N_{S}$ are stable they are coprime. Moreover defining an operator $S$ from

$$
S=\tilde{M} N_{S}-\tilde{N} M_{S}=\left(\tilde{M} G_{S}-\tilde{N}\right)\left(\tilde{V}-\tilde{U} G_{S}\right)^{-1}
$$

then under existence of the relevant inverse as in (2.16), $M_{S}, N_{S}$ can le characterized by a mapping on $S$ as

$$
\left[\begin{array}{l}
M_{S} \\
N_{S}
\end{array}\right]=\left[\begin{array}{cc}
\tilde{V} & -\tilde{U} \\
-\tilde{N} & \tilde{M}
\end{array}\right]^{-1}\left[\begin{array}{l}
I \\
S
\end{array}\right]
$$

Additionally, when $K$ "stabilizes" $G$ in the restricted sense of (2.17), then: $M_{S}, N_{S}$ will be stable iff $S$ is stable. Furthermore, (9.4)-(9.5) give a bijec. tion between the set of all plants $G_{S}$ such that (3.1) holds, and the set $0_{\text {: }}$ all operators $S$ such that

$$
M_{S}^{-1}=\left(\left[\begin{array}{ll}
I & 0
\end{array}\right]\left[\begin{array}{cc}
\tilde{V} & -\tilde{U} \\
-\tilde{N} & \tilde{M}
\end{array}\right]^{-1}\left[\begin{array}{l}
I \\
S
\end{array}\right]\right)^{-1} \quad \text { exists. }
$$

Proof: Note that under existence of $\left(\tilde{V}-\tilde{U} G_{S}\right)^{-1}$ we have

$$
G_{S}=G_{S}\left(\tilde{V}-\tilde{U} G_{S}\right)^{-1}\left(\tilde{V}-\tilde{U} G_{S}\right)=N_{S} M_{S}^{-1}
$$

Thus verifying (3.2). Now show (3.3)

$$
\begin{aligned}
\tilde{V} M_{S}-\tilde{U} N_{S} & =\tilde{V}\left(\tilde{V}-\tilde{U} G_{S}\right)^{-1}-\tilde{U} G\left(\tilde{V}-\tilde{U} G_{S}\right)^{-1} \\
& =\left(\tilde{V}-\tilde{U} G_{S}\right)\left(\tilde{V}-\tilde{U} G_{S}\right)^{-1}=I .
\end{aligned}
$$

Combining $G_{S}=N_{S} M_{S}^{-1}$ and (3.3) proves (3.4). Now note that

$$
\left[\begin{array}{cc}
\tilde{V} & -\tilde{U} \\
-\tilde{N} & \tilde{M}
\end{array}\right]\left[\begin{array}{c}
M_{S} \\
N_{S}
\end{array}\right]=\left[\begin{array}{c}
\tilde{V} M_{S}-\tilde{U} N_{S} \\
\tilde{M} N_{S}-\tilde{N} M_{S}
\end{array}\right]=\left[\begin{array}{l}
I \\
S
\end{array}\right]
$$

So under (2.16), (3.5) holds as claimed. 


\section{COPRIME FACTORIZATION ANALYSIS}

Under our assumptions, including (2.17) we have $\left[\begin{array}{cc}\tilde{V} & -\tilde{U} \\ -\tilde{N} & \tilde{M}\end{array}\right]$ i nimodular. Hence $(3.5)$ gives $\left[\begin{array}{l}M_{S} \\ N_{S}\end{array}\right]$ stable iff $\left[\begin{array}{l}I \\ S\end{array}\right]$ is stable. The identity mapping $I$ is trivially stable, hence $M_{S}, N_{S}$ are stable iff $S$ is stable.

Now let us prove bijectivity of the maps (3.4)-(3.5). It is evide it from the equations that given an $S$ such that (3.6) holds, $G_{S}=N_{S} \cdot V_{S}^{-1}$ is constructed from (3.5), and (3.1) holds. Similarly given $G_{S}$ such th th (3.1) holds, the $S$ obtained from (3.4) will satisfy (3.6), as $M_{S}=\left(\tilde{V} G_{S}-\tilde{J}\right)^{-1}$ is invertible. Hence the mapping from each class to the other is well cefined, and thus onto. To prove bijectivity it remains to prove that the images under the maps are unique.

Note that (3.5) shows that for each $S$ there exists a unique pair $M_{S}, N_{S}$.

$$
M_{S}^{-1}=\left(\left[\begin{array}{ll}
I & 0
\end{array}\right]\left[\begin{array}{cc}
\tilde{V} & -\tilde{U} \\
-\tilde{N} & \tilde{M}
\end{array}\right]^{-1}\left[\begin{array}{l}
I \\
S
\end{array}\right]\right)^{-1}
$$

so it is necessary that $S$ satisfy (3.6) for $M_{S}^{-1}$ to exist. Further the plant $G_{S}$ so obtained will satisfy (3.1). Hence the conditions (3.1) and (36) are equivalent. The bijectivity of the maps (3.4)-(3.5) is now establisherl.

The map from $S$ to $G$ as defined by (3.2) is onto (surjective), as for all $G$ such that (3.1) holds we have an $S$ as given by (3.4) which maps to $G$. To prove one to oneness (injectivity) consider that there exist $S_{1}$ and $S_{2}$ such that $G_{S_{1}}=G_{S_{2}}$. Then the $S^{\prime}$ 's of (3.4) are the same, giving

$$
\begin{aligned}
&\left(\tilde{M} N_{S_{1}}-\tilde{N} M_{S_{1}}\right)=\left(\tilde{M} N_{S_{2}}-\tilde{N} M_{S_{2}}\right) \\
& {\left[\begin{array}{cc}
\tilde{M} & -\tilde{N}
\end{array}\right]\left[\begin{array}{c}
N_{S_{1}} \\
M_{S_{1}}
\end{array}\right]=\left[\begin{array}{ll}
\tilde{M} & -\tilde{N}
\end{array}\right]\left[\begin{array}{c}
N_{S_{2}} \\
M_{S_{2}}
\end{array}\right] } \\
& {\left[\begin{array}{ll}
0 & I
\end{array}\right]\left[\begin{array}{cc}
\tilde{V} & -\tilde{U} \\
-\tilde{N} & \tilde{M}
\end{array}\right]\left[\begin{array}{cc}
\tilde{V} & -\tilde{U} \\
-\tilde{N} & \tilde{M}
\end{array}\right]^{-1}\left[\begin{array}{c}
I \\
S_{1}
\end{array}\right]=} \\
& {\left[\begin{array}{ll}
0 & I
\end{array}\right]\left[\begin{array}{cc}
\tilde{V} & -\tilde{U} \\
-\tilde{N} & \tilde{M}
\end{array}\right]\left[\begin{array}{cc}
\tilde{V} & -\tilde{U} \\
-\tilde{N} & \tilde{M}
\end{array}\right]^{-1}\left[\begin{array}{c}
I \\
S_{2}
\end{array}\right] } \\
& {\left[\begin{array}{ll}
0 & I
\end{array}\right]\left[\begin{array}{c}
I \\
S_{1}
\end{array}\right]=\left[\begin{array}{ll}
0 & I
\end{array}\right]\left[\begin{array}{c}
I \\
S_{2}
\end{array}\right] } \\
& S_{1}=S_{2}
\end{aligned}
$$

Hence the mapping is injective. This gives bijectivity of the maps, thus completing the proof. 


\section{A.D.B. PAICE, J.B. MOORE, AND R. HOROWITZ}

\section{Remarks}

1. A dual result to this involving right factorizations of $G$ and $K$, and giving a left factorization for $G_{S}$ is elusive at the moment, unless additional assumptions are made. However, dualizing in terms of interchanging the roles of $G$ and $S$ gives an expression for $S$ when $G_{S}$ is expressed in terms of $r c f^{\prime} s$ of $G, K$, as shown in the following theorem.

2. In the case that $(\tilde{V}-\tilde{U} G)^{-1}$ exists, then the theorem gives $G_{S}=G$ iff $S=0$. i.e, given a left factorization of $G, K$ we can get a rcf for $G$, also for $K$ as is shown in the dual to this theorem, Theorem 3.3.

3. In the linear $S$ case the expression for $G_{S}$ simplifies to give $G_{S}=$ $(\tilde{M}+S \tilde{U})^{-1}(\tilde{N}+S \tilde{V})$.

4. This theorem is of interest in the work done by Hammer [6], and by Tay and Moore [18]. In this work the plant $G$ is stabilized by a pre-, post-compensator pair $\tilde{V}^{-1}, \tilde{U}$, so that the question of well-posedness and stability of the system is reduced to that of the existence and stability of the operator $(\tilde{V}-\tilde{U} G)^{-1}$. This theorem shows that any plant $G_{S}$ for which this system is well posed is related to a nominal plant $G$ by means of (3.5), and is parameterized by the operator $S$. Furthermore, as $\left(\tilde{V}-\tilde{U} G_{S}\right)^{-1}=M_{S}$, the system is stable iff $S$ is stable. Thus the theorem gives the class of all plants stabilized by the pre-, post-compensator pair $\tilde{V}^{-1}, \tilde{U}$.

Theorem 3.2 Consider a well-posed and stable system $\{G, K\}$ with right coprime factorizations (2.2), (2.5), so that existence and stability conditions (2.7) and (2.8) hold. Consider a map S such that $(M-U S)^{-1}$ exists. Then $S$ has a right factorization $S=P_{G} D_{G}^{-1}$ given by

$$
\left[\begin{array}{l}
D_{G} \\
P_{G}
\end{array}\right]=\left[\begin{array}{c}
I \\
S
\end{array}\right](M-U S)^{-1} \text {, and } M D_{G}-U P_{G}=I \text {. }
$$

Further there exists a plant $G_{S}$ such that

$$
\begin{gathered}
G_{S}=N D_{G}-V P_{G}=(N-V S)(M-U S)^{-1} \\
{\left[\begin{array}{c}
D_{G} \\
P_{G}
\end{array}\right]=\left[\begin{array}{cc}
M & -U \\
-N & V
\end{array}\right]^{-1}\left[\begin{array}{c}
I \\
-G_{S}
\end{array}\right] .}
\end{gathered}
$$

Moreover this gives a bijection between the class of all operators $S$ such that $(M-U S)^{-1}$ exists and the class of all plants such that

$$
D_{G}^{-1}=\left(\left[\begin{array}{ll}
I & 0
\end{array}\right]\left[\begin{array}{cc}
M & -U \\
-N & V
\end{array}\right]^{-1}\left[\begin{array}{c}
I \\
-G_{S}
\end{array}\right]\right)^{-1} \text { exists. }
$$




\section{COPRIME FACTORIZATION ANALYSIS}

Proof: The details of the proof of this result are the same of thosi: of the previous theorem, as they are dual results, interchanging the roles of $S$ and $G$.

\section{Remarks}

1. In the case that the plant and controller, and their factoriz ations, are linear, the conditions (3.1) and (3.19) are equivalent, as as (3.6) and the existence of $(M+U S)^{-1}$. The theorems then give the same result.

2. These results may be readily dualized, interchanging the roles of the plant and controller.

3. Note that these theorems provide a natural setting for generating the class of all plants stabilized by the controller $K$, and in the dual case, the class of all controllers stabilizing a given plant. Theorem 3.1 may be applied to the main results of [15] to generate the class of all slants bounded-input stabilized by a given controller. By assuming linearity of $K$, it is possible to show that the class of all controllers stat ilized by $K$ can be generated by Theorem 3.2. More general resul s are elusive at this time, so that it is not possible to say whether $G$; will be stabilized by $K$.

\section{State Space Factorization Descriptions}

Consider now a state space approach to the factorization of nonlineas systems. Starting from a quite general state space description for a continuous time plant, stable right factorizations are developed, based on the assimption that the state equation of the plant is stabilizable by nonlinear state feedback. The development parallels the development of the theory for linear systems.

\section{Nonlinear System Class}

Given a real vector space $\mathcal{X}$, define the space of trajectories withir $\mathcal{X}$, $C(\mathcal{X})$ as in Section 2, first derivative. Any function which is contint ous and has continuous first derivative is called $\mathcal{C}^{1}$.

Consider that a plant $G\left(x_{0}\right): C(\mathcal{U}) \mapsto C(\mathcal{Y})$ is an operator, dependent on the parameter $x_{0}$, which maps inputs $u(\cdot)$ to outputs $y(\cdot)$. Furthermire, consider that $G\left(x_{0}\right)$ has state space description of the form

$$
G\left(x_{0}\right): \dot{x}=f(x, u), \quad y=h(x, u), \quad x(0)=x_{0} .
$$

A fundamental property of differential equations that we shall be exploiting is the existence and uniqueness of solutions of the differential equa- 


\section{A.D.B. PAICE, J.B. MOORE, AND R. HOROWITZ}

tion. The following theorem, adapted from [10], and stated without proof is useful.

Theorem 4.1 Let $f: \mathcal{X} \mapsto \mathcal{X}$ be a $\mathcal{C}^{1}$ map and let $x_{0} \in \mathcal{X}$. Then there exists a unique maximal open interval $(a, b)$ containing 0 , and a unique function $x:(a, b) \mapsto \mathcal{X}$ satisfying

$$
\dot{x}=f(x), \quad x(0)=x_{0} .
$$

\section{Remarks}

1. Note that $a, b$ may be equal to plus or minus infinity. In the case that $b$ is finite, the system is unstable, with finite escape time. Similarly if $a$ is finite, the reverse time system has finite escape time.

2. This theorem also gives results for the time varying case, and for systems of the form of (4.1), as is explored in the following corollaries.

Corollary 4.1 Let $f: \mathcal{X} \times \mathcal{R} \mapsto \mathcal{X}$ be a $\mathcal{C}^{1}$ map, and let $x_{0} \in \mathcal{X}$. Then there exists a unique maximal open interval $(a, b)$ containing 0 , and $a$ unique function $x:(a, b) \mapsto \mathcal{X}$ satisfying

$$
\dot{x}=f(x, t), \quad x(0)=x_{0} .
$$

Proof: Let $y=\left(\begin{array}{c}x \\ t\end{array}\right)$, and $g(y)=\left(\begin{array}{c}f(x, t) \\ 1\end{array}\right)$. As $f$ is $\mathcal{C}^{1}, g$ is $\mathcal{C}^{1}$, now apply Theorem 4.1 .

Corollary 4.2 Let $f: \mathcal{X} \times \mathcal{U} \mapsto \mathcal{X}$ be a $\mathcal{C}^{1}$ map, and let $x_{0} \in \mathcal{X}$. Then given $u \in C(\mathcal{U})$ there exists a unique maximal open interval $(a, b)$ containing 0 , and a unique function $x:(a, b) \mapsto \mathcal{X}$ satisfying

$$
\dot{x}=f(x, u(t)), \quad x(0)=x_{0} .
$$

Proof: Given $u \in C(\mathcal{U})$ set $g(x, t)=f(x, u(t))$ which is $\mathcal{C}^{1}$ as $f$ and $u$ are both $\mathcal{C}^{1}$, and apply the previous corollary.

In order to guarantee existence and uniqueness of solutions it is assumed that the map $f(\cdot, \cdot)$ of $(4.1)$ is $\mathcal{C}^{1}$. Unless otherwise stated all functions in the work to follow shall be assumed to be $\mathcal{C}^{1}$.

\section{Composition of Nonlinear Operators}

Consider two operators of the form of $(4.1), A\left(x_{0}\right): C(\mathcal{U}) \mapsto C(\mathcal{Y})$ and $B\left(v_{0}\right): C(\mathcal{Y}) \mapsto C(\mathcal{Z})$.

$$
\begin{array}{rrr}
A\left(x_{0}\right): \dot{x}=f_{A}(x, u), y=h_{A}(x, u), & x(0)=x_{0} \\
B\left(v_{0}\right): \dot{v}=f_{B}(v, y), \quad z=h_{B}(v, y), & v(0)=v_{0}
\end{array}
$$


Then the operator $C\left(x_{0}, v_{0}\right)=B\left(v_{0}\right) A\left(x_{0}\right): C(\mathcal{U}) \mapsto C(\mathcal{Z})$ will have state space description

$$
\begin{gathered}
C\left(x_{0}, v_{0}\right):\left(\begin{array}{c}
\dot{x} \\
\dot{v}
\end{array}\right)=\left(\begin{array}{c}
f_{A}(x, u) \\
f_{B}\left(v, h_{A}(x, u)\right)
\end{array}\right), \\
\left(\begin{array}{c}
x(0) \\
v(0)
\end{array}\right)=\left(\begin{array}{c}
x_{0} \\
v_{0}
\end{array}\right) \\
z=h_{B}\left(v, h_{A}(x, u)\right) .
\end{gathered}
$$

Note that in general the dimension of the state of $C\left(x_{0}, v_{0}\right)$ is equal to the sum of the dimensions of the states of $A\left(x_{0}\right)$ and $B\left(v_{0}\right)$. In some special cases, however, it may be possible to reduce the state, as shown in the following lemma.

Lemma 4.1 Consider operators $A\left(x_{0}\right), B\left(v_{0}\right)$ as given by (4.5), (4.6). Then if

$$
f_{B}\left(x, h_{A}(x, u)\right)=f_{A}(x, u), \quad x_{0}=v_{0}
$$

then $C\left(x_{0}, v_{0}\right)=C\left(x_{0}, v_{0}\right)=C\left(x_{0}\right)$ is reduced to the form

$$
C\left(x_{0}\right): \dot{x}=f_{A}(x, u) z=h_{B}\left(x, h_{A}(x, u)\right), \quad x(0)=x_{0} .
$$

Proof: Suppose that (4.9) hold, and consider the evolution of the state equations (4.5), (4.6). Substituting $v(t)=x(t)$ into (4.7) gives

$$
\begin{aligned}
\dot{v}(t)=f_{B}\left(v(t), h_{A}(x(t), u(t))\right) & =f_{B}\left(x(t), h_{A}(x(t), u(t))\right) \\
& =f_{A}(x(t), u(t))=\dot{x}(t) .
\end{aligned}
$$

Hence a solution of $(4.5),(4.6)$ is $v(t)=x(t), \forall t$. Note that $f_{B}, h_{A}, f_{A}$ are $\mathcal{C}^{1}$ functions. Hence by Theorem 4.1 this is the unique solution, and the lemma is established.

\section{Inverse Systems}

In deriving later results it will be necessary to be able to invert a nonlinear operator of the form of (4.1). The following lemma shows that given the existence of a map $h^{\#}: \mathcal{X} \times \mathcal{Y} \mapsto \mathcal{U}$, associated with the map $h$ of (4.1), it is possible to invert the operator. The map $h^{\#}(., \cdot)$ is called the pseudoinverse of $h(\cdot, \cdot)$, and the map $h(\cdot, \cdot)$ is called pseudo-invertible, this is an analogue of the reversible feedback function of [9]. Note that if $h(\cdot, \cdot)$ is $\mathcal{C}^{1}$, then $h^{\#}(\cdot, \cdot)$ is $\mathcal{C}^{1}$.

Lemma 4.2 Consider an operator $G\left(x_{0}\right)$ as in (4.1), construct the operator $S\left(x_{0}\right): C(\mathcal{Y}) \mapsto C(\mathcal{U})$ as follows.

$$
S\left(v_{0}\right): \dot{v}=f\left(v, h^{\#}(v, y)\right), \quad u=h^{\#}(v, y), \quad v(0)=v_{0} .
$$


Consider also that $h^{\#}$ satisfies

$$
h^{\#}(x, h(x, u))=u \quad \forall x, u
$$

then $S\left(x_{0}\right)$ is a left inverse for $G\left(x_{0}\right)$, i.e $S\left(x_{0}\right) G\left(x_{0}\right)=I$, and if $h^{\#}$ satisfies

$$
h\left(x, h^{\#}(x, u)\right)=u \quad \forall x, u
$$

then $S\left(x_{0}\right)$ is a right inverse for $G\left(x_{0}\right)$, i.e $G\left(x_{0}\right) S\left(x_{0}\right)=I$. Moreover, when $h$ and $h$ \# satisfy both (4.19), then (4.14), $S\left(x_{0}\right)$ is an inverse for $G\left(x_{0}\right)$, i.e

$$
G^{-1}\left(x_{0}\right)=S\left(x_{0}\right) .
$$

Proof: Consider the state equation of the composition $S\left(x_{0}\right) G\left(x_{0}\right)$

$$
\begin{array}{ccc}
\dot{x}= & f(x, u) & x(0)=x_{0} \\
\dot{v}= & f\left(v, h^{\#}(v, h(x, u))\right) & v(0)=x_{0}
\end{array}
$$

Note that by (4.13) we have for $x=v, f(x, u)=f\left(v, h^{\#}(v, h(x, u))\right)$. Applying Lemma 4.1 gives $x(t)=v(t), \forall t$. The output equation for $S\left(x_{0}\right) G\left(x_{0}\right)$ thus becomes

$$
z=h^{\#}(v, h(x, u))=u .
$$

This proves the first part of the lemma. A similar argument shows that when (4.14) is satisfied $G\left(x_{0}\right) S\left(x_{0}\right)=I$. This completes the proof.

Remark. Note the dependence on initial conditions for ensuring that the states remain equal for all time. This may be difficult to guarantee, additionally the error dynamics may be such that any small error is magnified. The question of when it is possible to stably invert a function, i.e. invert it and have the error dynamics such that any error will decrease with time, is a difficult one, and is dependent on the particular functions $f, h, h^{\#}$.

\section{Right Factorization for $G$}

The development of a stable right factorization for the plant $G\left(x_{0}\right)$ is critically dependent on the solution of the smooth state feedback stabilization problem for the state equation of $G\left(x_{0}\right)$. This is in itself an open problem, and a treatment of such is beyond the bounds of this paper. Here, the assumption is made that for plants of interest the stabilization problem has been solved and that the solution is available.

Consider the state feedback map $g(\cdot, \cdot): \mathcal{X} \times \mathcal{U} \mapsto \mathcal{U}$, as in Figure 2, so that the state equation for $G\left(x_{0}\right)$ becomes

$$
\dot{x}=f(x, g(x, u)), \quad x(0)=x_{0} .
$$




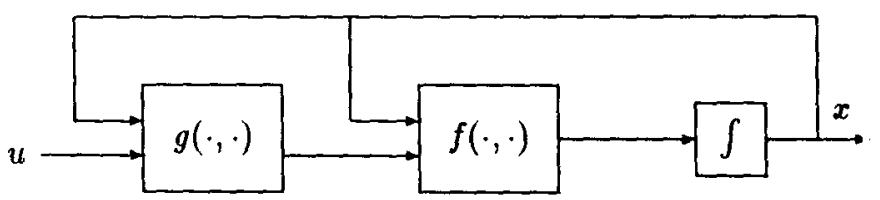

Figure 2: State feedback $g(\cdot, \cdot)$.

Assumption For the plant $G\left(x_{0}\right)$ of (4.1) there exists a pseudoinvertible $\mathcal{C}^{1}$ map $g(\cdot, \cdot): \mathcal{X} \times \mathcal{U} \mapsto \mathcal{U}$ such that the state equation (4.19) is stable, and $g^{\#}(\cdot, \cdot)$ satisfies both (4.13) and (4.14).

It is now possible to construct a stable right factorization for $G\left(x_{0}\right)$ as is explored in the following lemma. This lemma is equivalent to $T$ reorem 3 of [22], and parallels the discrete time results found in [8].

Lemma 4.3 Consider a plant $G\left(x_{0}\right)$ such that there exists a mal' $g(\cdot, \cdot)$ satisfying (4.20). Then it is possible to construct a stable right factorization for $G\left(x_{0}\right)$ as follows.

$$
\begin{aligned}
G\left(x_{0}\right)= & N\left(x_{0}\right) M^{-1}\left(x_{0}\right) \\
M\left(x_{0}\right): \dot{x}_{m}= & f\left(x_{m}, g\left(x_{m}, s\right)\right), \quad z=g\left(x_{m}, s\right), \\
& x_{m}(0)=x_{0} \\
N\left(x_{0}\right): \dot{x}_{n}= & f\left(x_{n}, g\left(x_{n}, s\right)\right), \quad y=h\left(x_{n}, g\left(x_{n}, s\right)\right) \\
& x_{n}(0)=x_{0}
\end{aligned}
$$

\section{Remarks}

1. By introducing the notion of detectability Verma [22] is able to prove that this a coprime factorization. The notion of detectability uised is that if $u$ and $y$ are stable, then $x$ is stable, and furthermore, thit for some $\beta>0,\|x\| \leq \beta\left\|\left[\begin{array}{l}y \\ u\end{array}\right]\right\|$.

2. Note that the equation $G\left(x_{0}\right)=N\left(x_{0}\right) M^{-1}\left(x_{0}\right)$ depends on th: initial conditions being identical, so that the Remarks following Lesnma 4.2 are appropriate here as well.

3. The requirement that $g(\cdot, \cdot)$ be pseudo-invertible is necessary fo: invertibility of $M\left(x_{0}\right)$. It does not appear overly restrictive, as ir. the linear case we have $g(x, u)=F x+u$, so that $g^{\#}(x, y)=y-F x$, where $\mathrm{F}$ is some matrix chosen such that $A+B F$ is stable. Furthermore, note that in [17], Sontag proves an input to state statility result which gives a stability result satisfying (4.20). Specifically it 


\section{A.D.B. PAICE, J.B. MOORE, AND R. HOROWITZ}

is shown that for systems of the form of (4.1), a feedback law of the form

$$
g(x, u)=K(x)+G(x) u
$$

where $G(x)$ is invertible for all $x$. In this case we have

$$
g^{\#}(x, v)=G(x)^{-1}(v-K(x))
$$

and the assumptions of (4.20) are satisfied.

\section{Factorizing a Universal Controller}

To illustrate the effectiveness of this approach to right factorization we give a right factorization of a universally stabilizing controller due to Nusbaum [13].

Lemma 4.4 Consider a first order SISO linear plant, with realization

$$
\begin{array}{rlr}
G: & \dot{x}=a x+b u & x(0)=x_{0} \\
y=x & .
\end{array}
$$

Where $b \neq 0$. Then there exists a nonlinear controller which will stabilize this plant for all values of $a$ and $b$. The state equations are:

$$
\begin{array}{rlr}
K: & \dot{v}=y\left(v^{2}+1\right) & v(0)=0 \\
& u=y\left(v^{2}+1\right) h(v) . &
\end{array}
$$

The proof of this lemma may be found in [13]. The function $h(\cdot)$ must satisfy certain conditions to ensure convergence, see [13] for details. Note that the function $h(x)=e^{x} \cos x$ satisfies these conditions.

Lemma 4.5 The controller $K$ of (4.27) has a pseudo-invertible stabilizing state feedback, $g(v, y)=y-v$, and thus has a stable right coprime factorization $K=U V^{-1}$ given by

$$
\begin{array}{lll}
U: & \dot{x_{u}}=\left(s-x_{u}\right)\left(x_{u}^{2}+1\right) & x_{u}(0)=0 \\
& u=s\left(x_{u}^{2}+1\right) h\left(x_{u}\right) & \\
V: & \dot{x_{v}}=\left(s-x_{v}\right)\left(x_{v}^{2}+1\right) & x_{v}(0)=0 \\
& y=s-x_{v} &
\end{array}
$$

Proof: It is straightforward to see that $g(., \cdot)$ is pseudo-invertible. It is now shown that $\dot{v}=(y-v)\left(v^{2}+1\right)$ is stable. First note that $\dot{v}=0$ iff $y=v$. If $v<y$, then $\dot{v}>0$, so $v$ will grow to converge to $y$. If $v>y$, then $\dot{v}<0$, so $v$ will converge down to $y$. Hence $v$ will track $y$, and so if $y$ is stable $v$ will be stable. Now apply lemma 4.3 to show that $U V^{-1}$ is a stable right factorization of $K$. Following the remarks we note that this is a $r c f$. 


\section{COPRIME FACTORIZATION ANALYSIS}

\section{Stabilizing Controller}

Following the linear theory a controller is designed based on the idea of a state estimator. Consider that there exists a map $l(\cdot, \cdot): \mathcal{X} \times\urcorner \vdash \mathcal{X}$, such that the state equation

$$
\dot{v}=f(v, u)-l(v, h(x, u)-h(v, u)) \quad v(0)=v_{0}
$$

acts as a state estimator for (4.1). i.e

Assumption For the plant $G\left(x_{0}\right)$ of (4.1) there exists a $\mathcal{C}^{1}$ map $l(\cdot, \cdot): \mathcal{X} \times \mathcal{Y} \mapsto \mathcal{X}$ such that (4.30) acts as a state estimator for $G\left(x_{0}\right)$, in that for all $x_{0}, v_{0}$, as $t \rightarrow \infty, v(t) \rightarrow x(t)$.

It is evident that $l(x, 0)=0, \forall x$, otherwise when $v(t)=x(t), \quad \dot{x}(t) \neq$ $\dot{x}(t)$. As in the previous case of the design of a stabilizing state ferdback map, the derivation of an $l(\cdot, \cdot)$ for a particular realization (4.1) is a:1 open problem, and as such is beyond the scope of this paper. The controller $K\left(x_{0}\right): C(\mathcal{Y}) \mapsto C(\mathcal{U})$ is then constructed as follows:

$$
\begin{gathered}
K\left(x_{0}\right): \dot{x}_{k}=\quad f\left(x_{k}, g\left(x_{k}, 0\right)\right)-l\left(x_{k}, y-h\left(x_{k}, g\left(x_{k}, 0\right)\right)\right), \\
x_{k}(0)=x_{0} \\
u=g\left(x_{k}, 0\right) .
\end{gathered}
$$

The stable right factorization $K=U V^{-1}$ is realizable with state space realizations

$$
\begin{array}{rlr}
V\left(v_{0}\right): \dot{x}_{v} & =f\left(x_{v}, g\left(x_{v}, 0\right)\right)-l\left(x_{v}, s\right), \\
z & =h\left(x_{v}, g\left(x_{v}, 0\right)\right)+s, \quad x_{v}(0)=v_{0} \\
U\left(u_{0}\right): \dot{x}_{u} & =f\left(x_{u}, g\left(x_{u}, 0\right)\right)-l\left(x_{u}, s\right), \\
u & =g\left(x_{u}, 0\right), & x_{u}(0)=u_{0}
\end{array}
$$

Coprimeness of these factorizations is shown via Lemma 2.2. Consider the inverse of the operator $\left[\begin{array}{cc}M & -U \\ -N & V\end{array}\right]$. First note that $M\left(x_{0}\right)$ and $\Lambda^{\prime}\left(x_{0}\right)$ have the same initial conditions, and the same state when driven fron the same input. Let us denote the identical states for these operators as $x_{m}$. Similarly $V\left(x_{0}\right)$ and $U\left(x_{0}\right)$ have identical states $x_{v}$.

$$
\begin{aligned}
& {\left[\begin{array}{cc}
M & -U \\
-N & V
\end{array}\right]\left(\begin{array}{c}
x_{0} \\
x_{0}
\end{array}\right):} \\
& \left(\begin{array}{c}
\dot{x}_{m} \\
\dot{x}_{v}
\end{array}\right)=\left(\begin{array}{c}
f\left(x_{m}, g\left(x_{m}, s_{1}\right)\right) \\
f\left(x_{v}, g\left(x_{v}, 0\right)\right)-l\left(x_{v}, s_{2}\right)
\end{array}\right),\left(\begin{array}{c}
x_{m}(0) \\
x_{v}(0)
\end{array}\right)=\left(\begin{array}{c}
x_{0} \\
x_{0}
\end{array}\right) \\
& \left(\begin{array}{c}
u \\
y
\end{array}\right)=\left(\begin{array}{c}
g\left(x_{m}, s_{1}\right)-g\left(x_{v}, 0\right) \\
s_{2}+h\left(x_{v}, g\left(x_{v}, 0\right)\right)-h\left(x_{m}, g\left(x_{m}, s_{1}\right)\right)
\end{array}\right)
\end{aligned}
$$


From Lemma 4.2, invertibility of this system follows if it is possible tc rearrange (4.37) to give $s_{1}, s_{2}$ in terms of $u, y$. Note that

$$
\begin{aligned}
& s_{1}=g^{\#}\left(x_{m}, u+g\left(x_{v}, 0\right)\right) \\
& s_{2}=y+h\left(x_{m}, u+g\left(x_{v}, 0\right)\right)-h\left(x_{v}, g\left(x_{v}, 0\right)\right) .
\end{aligned}
$$

Hence the operator is invertible and has state space realization given by

$$
\begin{aligned}
& {\left[\begin{array}{cc}
M & -U \\
-N & V
\end{array}\right]^{-1}\left(\begin{array}{c}
x_{0} \\
x_{0}
\end{array}\right):\left(\begin{array}{c}
\dot{v}_{m} \\
\dot{v}_{v}
\end{array}\right)=} \\
& \quad\left(\begin{array}{c}
f\left(v_{m}, u+g\left(v_{v}, 0\right)\right) \\
\left.f\left(v_{v}, g\left(v_{v}, 0\right)\right)-l\left(v_{v}, y+h\left(v_{m}, u+g\left(v_{v}, 0\right)\right)-h\left(v_{v}, g\left(v_{v}, 0\right)\right)\right)\right)
\end{array}\right.
\end{aligned}
$$

$$
\begin{aligned}
\left(\begin{array}{l}
s_{1} \\
s_{2}
\end{array}\right)= & \left(\begin{array}{c}
g^{\#}\left(v_{m}, u+g\left(v_{v}, 0\right)\right) \\
y+h\left(v_{m}, u+g\left(v_{v}, 0\right)\right)-h\left(v_{v}, g\left(v_{v}, 0\right)\right)
\end{array}\right), \\
& \left(\begin{array}{c}
v_{m}(0) \\
v_{v}(0)
\end{array}\right)=\left(\begin{array}{c}
x_{0} \\
x_{0}
\end{array}\right) .
\end{aligned}
$$

Note that for $u=y=0$ and $v_{m}(0)=v_{v}(0), \dot{v}_{m}=\dot{v}_{v}=f\left(v_{v}, g\left(v_{v}, 0\right)\right)$, which is stable. In the case $u \neq 0, y \neq 0$ it is not as clear that (4.40) will remain stable, although the assumption (4.31) implies that the state $v_{v}$ will mimic $v_{m}$, giving stability. In [16] and [17] it is proven that if a system may be stabilized by state feedback so that for the zero input case the system is stable, then the system may also be input to state stabilized. Hence in this case it would seem that (4.40) will remain stable in the case $u \neq 0, y \neq 0$, at least for bounded $u, y$, however precise results are elusive at this time.

If this inverse operator $(4.40),(4.41)$ is stable, Lemma 2.2 and Theorem 2.1 can be applied to give coprimeness of the factorizations and stability of the system $\left\{G\left(x_{0}\right), K\left(x_{0}\right)\right\}$.

These results are summarized in the following lemma.

Lemma 4.6 Consider a plant $G\left(x_{0}\right)$, with state space description (4.1), such that there exist mappings $g(\cdot, \cdot)$, and $l(\cdot, \cdot)$ satisfying (4.20) and (4.31) respectively. Then there exists a controller $K\left(x_{0}\right)$ given by (4.32), (4.33), and such that the system $\left\{G\left(x_{0}\right), K\left(x_{0}\right)\right\}$ is stable. Furthermore, the right factorizations of $G\left(x_{0}\right)$ and $K\left(x_{0}\right)$ given by (4.22), (4.23), (4.34), (4.35) are coprime.

Remark. Note that this is only one possible approach to the stabilization of nonlinear systems, albeit the one which is the most fruitful if we are to take advantage of (4.20). 


\section{COPRIME FACTORIZATION ANALYSIS}

\section{Left Coprime Factorizations}

With the formulation of the plant $G\left(x_{0}\right)$ as in (4.1) it does not appear possible to generate stable left factorizations in the form of $(2.3)$. In the linear theory the construction of the left factorizations is critically dependent on being able to additively decompose the state of $G\left(x_{0}\right)$ into it's stable and unstable parts. The state of $\tilde{N}, x_{n}$ is the stable part of the state of $G, x$. The state of $\tilde{M}^{-1}, x_{m}$ models the difference between these, giving $x=x_{n}+x_{m}$ as is shown in the following equations.

$$
\begin{aligned}
\tilde{N} \quad: & \dot{x}_{n}=A x_{n}+B u+H\left(C x_{n}+D u\right) \\
& s=C x_{n}+D u \\
\tilde{M}^{-1} \quad: & \dot{x}_{m}=A x_{m}-H s=A x_{m}-H\left(C x_{n}+D u\right) \\
G \quad: & \dot{x}=\dot{x}_{n}+\dot{x}_{m}=A\left(x_{n}+x_{m}\right)+B u=A x+B u(
\end{aligned}
$$

A nonlinear analog of this process is not possible in the framework developed in this paper. Other attempts have been more successful. In [12] lcf's were obtained by using a specialized version of (4.1). This approach is explored in a following section.

\section{Stabilization of Other Plants}

A key objective of this paper can now be achieved by appropriate application of Theorem 3.2. Thus consider the problem of stabilizing a plant $G\left(x_{1}\right)$, where the initial condition $x_{1}$ is different from the initial condition $x_{0}$ of the controller $K\left(x_{0}\right)$ and the "idealized" nominal plant $G\left(x_{0}\right)$. It is then known that the system $\left\{G\left(x_{0}\right), K\left(x_{0}\right)\right\}$ is stable, but what about $\left\{G\left(x_{1}\right), K\left(x_{0}\right)\right\}$ ? A coprime factorization for $G\left(x_{1}\right)$ is $N\left(x_{1}\right) M^{-1}\left(x_{1}\right)$, and by Theorem 2.1 it is straightforward to see that the $\left\{G\left(x_{1}\right), K\left(x_{0}\right)\right\}$ is stable if the operator $\left[\begin{array}{cc}M & -U \\ -N & V\end{array}\right]^{-1}\left(\begin{array}{l}x_{1} \\ x_{0}\end{array}\right)$ is stable, but this is likely to be difficult to prove. The difference between the nominal plant $G\left(x_{0}\right)$ and the actual plant $G\left(x_{1}\right)$ can be parameterized by the map $S\left(x_{0}, x_{1}\right)$, of Theorem 3.2. From (3.18) and $S=P_{G} D_{G}^{-1}$, the following expression is obtained

$$
\begin{array}{rlr}
S\left(x_{0}, x_{1}\right) & & \\
\dot{w}= & f\left(w, g\left(w_{m}, r_{1}\right)+g\left(w_{v}, 0\right)\right) & w(0)=x_{1} \\
\dot{w}_{m}= & f\left(w_{m}, g\left(w_{m}, r_{1}\right)\right) & w_{m}(0)=x_{0} \\
\dot{w}_{v}= & f\left(w_{v}, g\left(w_{v}, 0\right)\right)-l\left(w_{v}, h\left(w, g\left(w_{m}, r_{1}\right)+\right.\right. & w_{v}(0)=x_{0} \\
& & \left.\left.g\left(w_{v}, 0\right)\right)-h\left(w_{v}, g\left(w_{v}, 0\right)\right)-h\left(w_{m}, g\left(w_{m}, r_{1}\right)\right)\right) \\
r_{2}= & h\left(w, g\left(w_{m}, r_{1}\right)+g\left(w_{v}, 0\right)\right)- & \\
& h\left(w_{v}, g\left(w_{v}, 0\right)\right)-h\left(w_{m}, g\left(w_{m}, r_{1}\right)\right) &
\end{array}
$$

The following identity now holds

$$
G\left(x_{1}\right)=\left(N\left(x_{0}\right)+V\left(x_{0}\right) S\left(x_{0}, x_{1}\right)\right)\left(M\left(x_{0}\right)+U\left(x_{0}\right) S\left(x_{0}, x_{1}\right)\right)^{-1} .
$$


Thus the effect of the different initial conditions may be modeled by the additional inputs $V\left(x_{0}\right) S\left(x_{0}, x_{1}\right)$, and $U\left(x_{0}\right) S\left(x_{0}, x_{1}\right)$.

Note that the application of Theorem 3.2 is not limited to this case. It is possible to find an $S\left(x_{0}, x_{1}\right)$ for any plant $G_{s}\left(x_{1}\right)$ for which the operal or $D_{G}^{-1}$ given by (3.19) exists.

\section{Left Factorizations}

In this section a restricted form of (4.1) is given, and it is shown how th is leads to a more complete factorization theory than that developed in $t$. 1 previous sections. These results are presented without proof, further deta is may be found in the companion paper by Moore and Irlicht [12].

Consider that the operator $G\left(x_{0}\right)$ has state space description given ty

$$
G\left(x_{0}\right): \quad \begin{aligned}
& \dot{x}=A(x) x+B(x) u \\
& y=C(x) x+D(x) u
\end{aligned} \quad x(0)=x_{0} .
$$

Then the existence of a map $g(\cdot, \cdot)=F(x) x+u$ satisfying (4.20) gives a stable right factorization as developed in Section 4 . The exact form $s$ of $M\left(x_{0}\right)$ and $N\left(x_{0}\right)$ follow from the definitions given, and may be found in Section 2 of [12]. Further if there exists a function $l(\cdot, \cdot)=H(x) y$ satisfying (4.31), it is possible to construct a controller $K\left(x_{0}\right)$, with stable right factorization $U\left(x_{0}\right) V\left(x_{0}\right)^{-1}$.

Working with this form of (4.1) is instructive as it illustrates mor? clearly how the linear theory generalizes to the results presented in thi; paper. Although further results do not appear attainable in this frame. work, it does present a natural setting for the development of left coprim: factorizations, and thus a more complete factorization theory.

Consider the generalization of (5.1) where there is an external signa. $y_{w}$ which is injected into each of the matrices $A(\cdot), B(\cdot), C(\cdot), D(\cdot)$ as follows:

$$
G_{w}\left(x_{0}\right): \quad \begin{aligned}
& \dot{x}=A\left(y_{w}\right) x+B\left(y_{w}\right) u \\
& y=C\left(y_{w}\right) x+D\left(y_{w}\right) u
\end{aligned} \quad x(0)=x_{0}
$$

Here $y_{w}$ is a signal which is generated by a strictly causal filter $W\left(w_{0}\right)$ acting on $y$, or $u$. Then by constructing the matrices $F\left(y_{w}\right), H\left(y_{w}\right)$ such that $A\left(y_{w}\right)+B\left(y_{w}\right) F\left(y_{w}\right)$ and $A\left(y_{w}\right)+H\left(y_{w}\right) C\left(y_{w}\right)$ are stable for all $y_{w}$, it is possible to construct stable right and left factorizations for $G_{w}\left(x_{0}\right)$ as follows:

$$
\begin{aligned}
& G_{w}\left(x_{0}\right)=N\left(x_{0}\right) M\left(x_{0}\right)^{-1} \\
& \dot{x}_{m}=\left(A\left(y_{w}\right)+B\left(y_{w}\right) F\left(y_{w}\right)\right) x_{m}+B\left(y_{w}\right) s_{r} \\
& M\left(x_{0}\right): u=F\left(y_{w}\right) x_{m}+s_{r} \\
& x_{m}(0)=x_{0}
\end{aligned}
$$




\section{COPRIME FACTORIZATION ANALYSIS}

$$
\begin{aligned}
& \dot{x}_{n}=\left(A\left(y_{w}\right)+B\left(y_{w}\right) F\left(y_{w}\right)\right) x_{n}+B\left(y_{w}\right) s_{r} \\
& \begin{array}{ll}
N\left(x_{0}\right): & y \\
x_{n}(0) & =\left(C\left(y_{w}\right)+D\left(y_{w}\right) F\left(y_{w}\right)\right) x_{n}+D\left(y_{w}\right) s_{r}
\end{array} \\
& G_{w}\left(x_{0}\right)=\tilde{M}\left(x_{0}\right)^{-1} \tilde{N}\left(x_{0}\right) \\
& \dot{x}_{\tilde{N}}=A\left(y_{w}\right) x_{\tilde{N}}+B\left(y_{w}\right) u+H\left(y_{w}\right)\left(C\left(y_{w}\right) x_{\dot{N}}+\right. \\
& \begin{array}{ll}
\tilde{N}\left(x_{0}\right): & \left.D\left(y_{w}\right) u\right) \\
& s_{1}=C\left(y_{w}\right) x_{N}+D\left(y_{w}\right) u
\end{array} \\
& x_{\hat{N}}(0)=x_{0} / 2 \\
& \begin{aligned}
\dot{x}_{\tilde{M}} & =\left(A\left(y_{w}\right)+H\left(y_{w}\right) C\left(y_{w}\right)\right) x_{\dot{M}}-H\left(y_{w}\right) y \\
s_{2} & =-C\left(y_{w}\right) x_{\tilde{M}}+y
\end{aligned} \\
& x_{\hat{N}}(0)=x_{0} / 2
\end{aligned}
$$

Note that as $A(\cdot), B(\cdot), C(\cdot), D(\cdot)$ are matrices it is possible to add the states of $\tilde{M}^{-1}$ and $\tilde{N}$ to get the state of $G$, i.e $x=v_{\tilde{M}}+x_{\tilde{N}}$, where $v_{\dot{M}}$ is the state of $\tilde{M}^{-1}$. The choice $x_{\tilde{M}}\left(x_{0}\right)=x_{0} / 2$ is somewhat arbitrary since $G\left(x_{0}\right)=\tilde{M}\left(m_{0}\right)^{-1} \tilde{N}\left(n_{0}\right)$ for all $m_{0}, n_{0}$ such that $m_{0} / 2+n_{0} / 2=x_{0}$.

A controller $K\left(x_{0}\right)$ may be constructed, having left and right factorizations as follows:

$$
\begin{aligned}
& \dot{x}_{k}=\left(A\left(y_{w}\right)+B\left(y_{w}\right) F\left(y_{w}\right)\right) x_{k}- \\
& \begin{aligned}
K_{w}\left(x_{0}\right): \quad & H\left(y_{w}\right)\left(y-\left(C\left(y_{w}\right)+D\left(y_{w}\right) F\left(y_{w}\right)\right) x_{k}\right) \\
u & =F\left(y_{w}\right) x_{k} \\
x_{k}(0)= & x_{0}
\end{aligned} \\
& K\left(x_{0}\right)=U\left(x_{0}\right) V\left(x_{0}\right)^{-1} \\
& \dot{x}_{v}=\left(A\left(y_{w}\right)+B\left(y_{w}\right) F\left(y_{w}\right)\right) x_{v}+B\left(y_{w}\right) s_{l} \\
& \begin{aligned}
V\left(x_{0}\right): \quad \begin{array}{l}
u \\
x_{v}(0)
\end{array}=\left(C\left(y_{w}\right)+D\left(y_{w}\right) F\left(y_{w}\right)\right) x_{v}+s_{l} \\
\end{aligned} \\
& \begin{array}{ll}
\dot{x}_{u} & =\left(A\left(y_{w}\right)+B\left(y_{w}\right) F\left(y_{w}\right)\right) x_{u}+B\left(y_{w}\right) s_{l} \\
y\left(x_{0}\right): \quad & =F\left(y_{w}\right) x_{u} \\
y & x_{u}(0)=x_{0}
\end{array} \\
& K\left(x_{0}\right)=\tilde{V}\left(x_{0}\right)^{-1} \tilde{U}\left(x_{0}\right)
\end{aligned}
$$

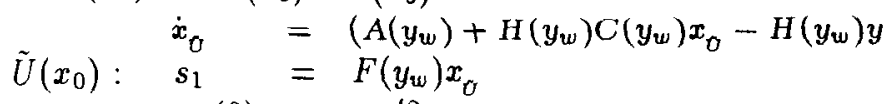

$$
\begin{aligned}
& x_{0}(0)=x_{0} / 2
\end{aligned}
$$




$$
\begin{aligned}
\dot{x}_{v}= & \left(A\left(y_{w}\right)+H\left(y_{w}\right) C\left(y_{w}\right)\right) x_{v}+\left(B\left(y_{w}\right)+\right. \\
\tilde{V}\left(x_{0}\right): \quad & \left.H\left(y_{w}\right) D\left(y_{w}\right)\right) u \\
s_{2}= & -F\left(y_{w}\right) x_{v}+u \\
x_{\tilde{v}}(0)= & x_{0} / 2
\end{aligned}
$$

As in Section 4 when there are no external inputs to the system, and the initial conditions of the plant and controller are the same, the state of $K_{w}\left(x_{0}\right)$ will track that of $G_{w}\left(x_{0}\right)$ giving stability of the system

$$
\left\{G_{w}\left(x_{0}\right) K_{w}\left(x_{0}\right)\right\} \text {. }
$$

The problem then addressed is how to construct the signal $y_{w}$ such that it may be included in the fractional descriptions of the plant and control er in a natural way. By making $y_{w}$ a function of the output $y$ of $G\left(x_{0}\right)$, it is possible to construct right and left factorizations for $G\left(x_{0}\right)$ and $K\left(x_{1}\right)$, however difficulties are encountered in trying to derive left factorizatio is of the controller which satisfy the Bezout identity $\tilde{V} M-\tilde{U} N=I$. n fact it is shown that with this particular formulation it is not possible :o construct a left factorization which satisfies this Bezout identity. To ove:come this problem the notion of an augmented plant $\mathcal{G}\left(x_{0}\right)=\left[G\left(x_{0}\right)^{\prime} l\right]^{\prime}$ is introduced. The state of $G\left(x_{0}\right)$ is used to construct the signal $y_{w}$ is follows:

$$
W\left(x_{0}\right): \quad \begin{aligned}
& \dot{x}_{w}=A\left(x_{w}\right) x_{w}+B\left(x_{w}\right) u \quad x_{w}(0)=x_{0} . \\
& y_{w}=x_{w}
\end{aligned}
$$

Hence $G\left(x_{0}\right)=G_{w}\left(x_{0}\right)$ and the right factorizations derived for $G\left(x_{0}\right)$ ar: equal to those of $G_{w}\left(x_{0}\right)$. The unity feedthrough term of the augmenterl plant ensures that the input to $G\left(x_{0}\right)$ is available to the controller $\mathcal{K}\left(x_{0}\right.$. which is constructed as follows.

$$
\begin{array}{rlr} 
& \mathcal{K}\left(x_{0}\right): C(\mathcal{Y}) \times C(\mathcal{U}) \mapsto C(\mathcal{U}) & \left(\begin{array}{l}
y \\
u
\end{array}\right) \mapsto u_{k} \\
\dot{x}_{w}=A\left(x_{w}\right) x_{w}+B\left(x_{w}\right) u & x_{w}(0)=x_{0} \\
\dot{x}_{k}=\left(A\left(x_{w}\right)+B\left(x_{w}\right) F\left(x_{w}\right)\right) x_{k}- & \\
u_{k}=F\left(x_{w}\right)\left(y-\left(C\left(x_{w}\right)+D\left(x_{w}\right) F\left(x_{w}\right)\right) x_{k}\right) & x_{k}(0)=x_{0} \\
& &
\end{array}
$$

Note that the state $x_{w}$ is the same as that of $W\left(x_{0}\right)$, so that the signal fed into the matrices $A(\cdot), \ldots, D(\cdot)$ in (5.3) and (5.2) are the same. It is shown that if the system $\left\{\mathcal{G}\left(x_{0}\right), \mathcal{K}\left(x_{0}\right)\right\}$ is bounded-input stable, then there exists a controller $\overline{\mathcal{K}}\left(x_{0}\right)$ such that the system $\left\{G\left(x_{0}\right), \overline{\mathcal{K}}\left(x_{0}\right)\right\}$ is bounded-input stable.

Factorizations for the augmented plant and controller may now be constructed. The plant factorizations may be given in terms of the previous factorizations, recalling that the signal $y_{w}$ is given by $(5.4)$, as follows. 


$$
\begin{aligned}
& \mathcal{G}\left(x_{0}\right)=\left[\begin{array}{c}
G\left(x_{0}\right) \\
I
\end{array}\right]=\mathcal{N}\left(x_{0}\right) \mathcal{M}\left(x_{0}\right)^{-1}=\tilde{\mathcal{M}}\left(x_{0}\right)^{-1} \tilde{\mathcal{N}}\left(x_{0}\right) \\
& \mathcal{M}\left(x_{0}\right)=M\left(x_{0}\right) \\
& \tilde{\mathcal{N}}\left(x_{0}\right)=\left[\begin{array}{c}
\tilde{N}\left(x_{0}\right) \\
I
\end{array}\right] \\
& \begin{aligned}
\mathcal{N}\left(x_{0}\right) & =\left[\begin{array}{c}
N\left(x_{0}\right) \\
M\left(x_{0}\right)
\end{array}\right] \\
\tilde{\mathcal{M}}\left(x_{0}\right) & =\left[\begin{array}{cc}
\tilde{M}\left(x_{0}\right) & 0 \\
0 & I
\end{array}\right]
\end{aligned}
\end{aligned}
$$

A left factorization for the controller may be written

$$
\begin{aligned}
& \mathcal{K}\left(x_{0}\right)=\tilde{\mathcal{V}}\left(x_{0}\right)^{-1} \tilde{\mathcal{U}}\left(x_{0}\right) \\
& \dot{x}_{w}=A\left(x_{w}\right) x_{w}+B\left(x_{w}\right) u \\
& \dot{x}_{v} \quad=\left(A\left(x_{w}\right)+H\left(x_{w}\right) C\left(x_{w}\right)\right) x_{v}+ \\
& \tilde{\mathcal{V}}\left(x_{0}\right): \quad\left(B\left(x_{w}\right)+H\left(x_{w}\right) D\left(x_{w}\right)\right) u \\
& s_{2} \quad=-F\left(x_{w}\right) x_{\hat{v}}+u \\
& x_{w}=x_{0} \\
& x_{\mathfrak{v}}(0)=x_{0} / 2 \\
& \dot{x}_{u}=A\left(x_{w}\right) x_{w}+B\left(x_{w}\right) u \\
& \dot{x}_{\hat{v}}=\left(A\left(x_{w}\right)+H\left(x_{w}\right) C\left(x_{w}\right)\right) x_{o}-H\left(x_{w}\right) y \\
& \tilde{U}\left(x_{0}\right): s_{1}=F\left(x_{w}\right) x_{\tilde{U}} \\
& x_{w}=x_{0} \\
& x_{0}(0)=x_{0} / 2
\end{aligned}
$$

The main result of [15] may now be applied giving the class of all stabilizing plants and controllers as follows.

Theorem 5.1 Consider the system $\left\{\mathcal{G}_{\mathcal{S}}\left(x_{0}, s_{0}\right), \mathcal{K}_{\mathcal{Q}}\left(x_{0}, q_{0}\right)\right\}$ as shown in Figure 3 , where $\tilde{\mathcal{M}}\left(x_{0}\right), \tilde{\mathcal{N}}\left(x_{0}\right), \tilde{\mathcal{U}}\left(x_{0}\right), \tilde{\mathcal{V}}\left(x_{0}\right)$ are lcf's of $\mathcal{G}\left(x_{0}\right), \mathcal{K}\left(x_{0}\right)$ which are differentially bounded. Consider also that

$$
\left[\begin{array}{cc}
\tilde{\mathcal{M}}\left(x_{0}\right) & -\tilde{\mathcal{N}}\left(x_{0}\right) \\
-\tilde{\mathcal{U}}\left(x_{0}\right) & \tilde{\mathcal{V}}\left(x_{0}\right)
\end{array}\right]^{-1}
$$

is BIBO stable. Then the system is $\min \left\{\varepsilon_{v}, \varepsilon_{N}\right\}, \min \left\{\varepsilon_{\sigma}, \varepsilon_{\dot{M}}\right\}$ bounded input stable iff the system $\left\{\mathcal{S}\left(s_{0}\right), \mathcal{Q}\left(q_{0}\right)\right\}$, of Figure 4 is $\left(\theta_{0}+\theta_{V}\right),\left(\theta_{\dot{M}}+t_{N}\right)$ bounded input stable.

Remark. Note that the relationship between $\mathcal{G}_{\mathcal{S}}\left(s_{0}, q_{0}\right)$ and $\mathcal{S}\left(s_{0}\right)$ is that described by Theorem 3.1. Dualizing this theorem in terms of intercha 1 ing the role of the plant and controller gives the relationship betwien $\mathcal{K}_{\mathcal{Q}}\left(x_{0}, q_{0}\right)$ and $\mathcal{Q}\left(q_{0}\right)$. 


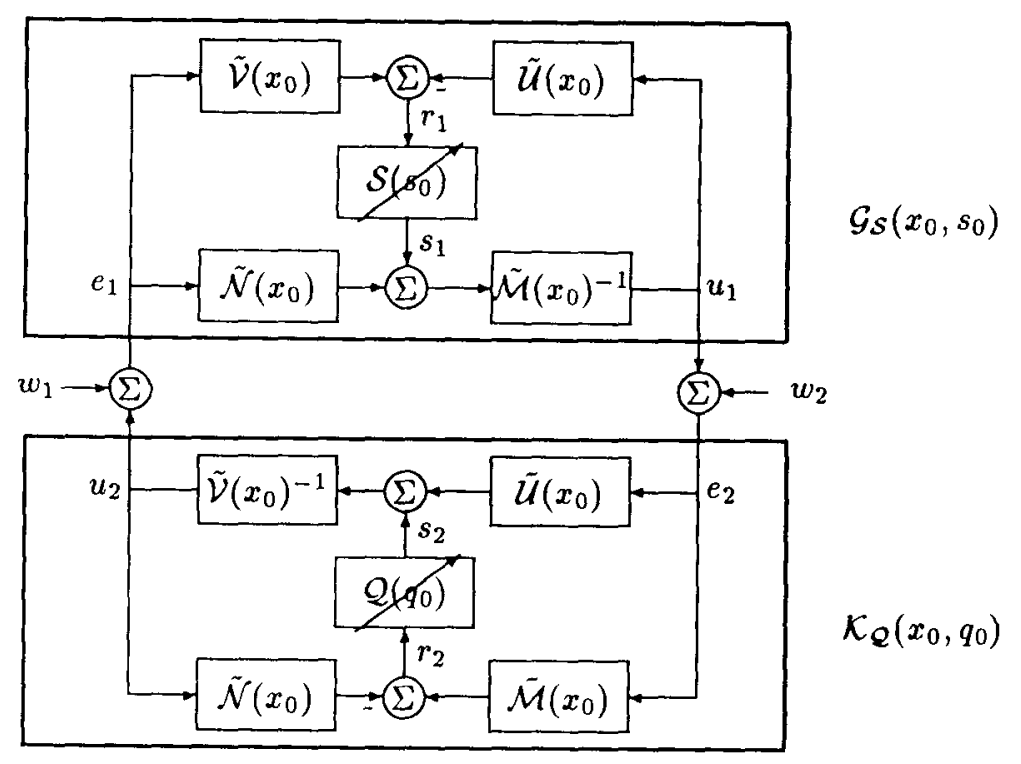

Figure 3: The feedback system $\left\{\mathcal{G}_{\mathcal{S}}\left(s_{0}, q_{0}\right), \mathcal{K}_{\mathcal{Q}}\left(x_{0}, q_{0}\right)\right\}$.

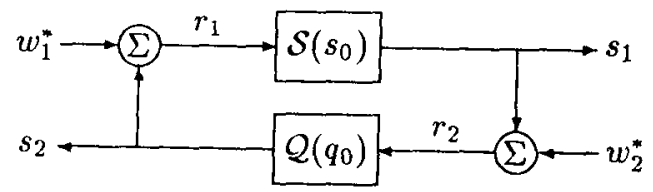

Figure 4: The feedback system $\left\{\mathcal{S}\left(s_{0}\right), \mathcal{Q}\left(q_{0}\right)\right\}$. 


\section{COPRIME FACTORIZATION ANALYSIS}

\section{Conclusion}

In this paper a quite general approach to the stabilization of nonlinear systems has been presented. The results given in the first two sections, considering the factorization approach from an input-output point of riew represent the most general formulation currently available. To obtain further results paralleling the linear theory, it is necessary to make simplifying assumptions, restricting the class of plants or controllers allowable.

The state-space approach presented considers the most general formulation possible, and is of interest mainly due to the way that it illuminates the properties of the dynamical systems that are necessary to generate results similar to those of the linear systems. Specifically the need for a stabili:ing state feedback map, and a stable state estimator for the realization of the plant is emphasized.

The final section presents a specialization of the work which generites classes of bounded input stabilizing plants and controllers. This shows the means by which the general input-output results currently available r ay be applied to the stabilization of nonlinear systems.

\section{References}

[1] C. A. Desoer. Right coprime factorizations of a class of time-varying nonlinear systems. Technical Report, Electronics Research Laboratcry, University of California, 1987.

[2] B. A. Francis. A Course in $H_{\infty}$ Control Theory. New York: Springar, 1987.

[3] J. Hammer. Nonlinear systems, stability and rationality, Internation al Journal of Control 40(1) (1984), 1-35.

[4] J. Hammer. Nonlinear systems, additive feedback and rationality, International Journal of Control 40 (November 1984), 953-969.

[5] J. Hammer. Nonlinear systems stabilization and coprimeness, Inter. national Journal of Control 42 (July 1985), 1-20.

[6] J. Hammer. Stabilization of nonlinear systems, International Journ ll of Control, 44 (November 1986), 1349-1381.

[7] J. Hammer. Fraction representations of nonlinear systems: A simplified approach, International Journal of Control 46 (1987), 455-472.

[8] J. Hammer. Fraction representations of non-linear systems and noitadditive state feedback, International Journal of Control 50 (1989), 1981-1990. 
[9] J. Hammer. State feedback for non-linear control systems, Inter zational Journal of Control 50 (1989), 1981-1990.

[10] M. W. Hirsch and S. Smale. Differential Equations, Dynamical Systems, and Linear Algebra. New York: Academic Press, 1984.

[11] A. J. Krener and Y. Zhu. The fractional representation of a class of nonlinear systems, Proc. of the 28th CDC (1989), 963-968.

[12] J. B. Moore and L. Irlicht. Coprime factorization over a class of nc nlinear systems, submitted for publication, 1991.

[13] R. D. Nussbaum. Some remarks on a conjecture in adaptive control, Systems and Control Letters 3 (1983), 243-246.

[14] A. D. B. Paice and J. B. Moore. On the Youla-Kucera parameterization for nonlinear systems, Systems and Control Letters 14 (1990), 12:129.

[15] A. D. B. Paice and J. B. Moore. Robust stabilization of nonline: .r plants via left coprime factorizations, Systems and Control Letters 15 (1990), 125-135.

[16] E. D. Sontag. Smooth stabilization implies coprime factorizatior, IEEE Transactions on Automatic Control 34 (1989), 435-443.

[17] E. D. Sontag. Further facts about input to state stabilization, IEEi? Transactions on Automatic Control 35 (1990), 473-476.

[18] T. T. Tay and J. B. Moore. Left coprime factorizations and a class $0^{\circ}$ stabilizing controllers for nonlinear systerns, International Journal $o_{4}^{\text {: }}$ Control 49 (1989), 1235-1248.

[19] T. T. Tay, J. B. Moore, and R. Horowitz. Indirect adaptive techniques for fixed controller performance enhancement, International Journal of Control 5 (1989), 1941-1959.

[20] M. S. Verma. Coprime fractional representations of nonlinear systems, Proc. IEEE Int. Symp. on Circuits and Systems 3 (1988), 2449.

[21] M. S. Verma. Coprime fractional representations and stability of nonlinear feedback systems, International Journal of Control 48 (1988), $897-918$.

[22] M. S. Verma and T. Pham. Stabilization of nonlinear systems and coprime factorization, Proc Int. Symp. MTNS-89 2 (1989), 473-482.

[23] M. Vidyasagar. Control System Synthesis: A Factorization Approach. Cambridge: MIT Press, Cambridge, 1985. 


\section{COPRIME FACTORIZATION ANALYSIS}

Department of Systems Engineering, Research School of Physical Sciences, Australian National University, GPO Box 4, CanBERRa ACT 2601, AUSTRalia

Department of Systems Engineering, Research School of JHyslCal Sciences, Australian National University, GPO Box 4 CanBERRa ACT 2601, AUSTRalia

Department of Mechanical Enginfering, University of Ca: IforNiA AT Berkeley, CA 94720, USA

Communicated by Clyde F. Martin 